\title{
Perturbative vs non-perturbative renormalization: the case of the quark mass
}

\section{Michele Brambilla}

University of Parma and INFN

E-mail: michele.brambilla@fis.unipr.it

\section{Francesco Di Renzo*}

University of Parma and INFN

E-mail: francesco.direnzo@is.unipr.it

\section{Luigi Scorzato}

$E C T^{*}$

E-mail: scorzatodect.it

\begin{abstract}
Comparing perturbative and non-perturbative results for renormalization constants has been an issue for a while. The quark mass renormalization constant is a prototype example: discrepancies between different results have been several times ascribed to this issue. Given the logarithmic nature of the divergence, there is no theoretical obstruction to a perturbative computation. The problem, as it is obvious, is how to perform the computation at high loops. Truncation errors should in turn be compared to a variety of errors (e.g. irrelevant effects, chiral extrapolation, finite size) which should be carefully assessed as well. We discuss the status of our computations in Numerical Stochastic Perturbation Theory, in particular for the tree level Symanzik improved gauge action at $n_{f}=2$. The emphasis is on main goal: how to take all the systematic effects under control at three loop level.
\end{abstract}

The XXVIII International Symposium on Lattice Field Theory

June 14-19,2010

Villasimius, Sardinia Italy

\footnotetext{
${ }^{*}$ Speaker.
} 


\section{Motivations}

The lattice community regards by now a percent accuracy on the computation of many observables as a realistic goal: we have entered the era of precision LGT computations. This of course requires that all the systematic effects are well under control. With this respect, comparing perturbative and non-perturbative results for renormalization constants has been an issue for a while. There are cases in which power divergences do not show up: for logarithmic divergences there is no theoretical obstruction to a perturbative computation, but this is only useful provided one can perform the computation at high loops [1]. The quark mass renormalization constant is a prototype example: discrepancies between different results have been several times ascribed to the issue of perturbative vs non-perturbative computation of the relevant renormalization constants [2].

Our group has been involved for a while in a project aiming at high loops computations of renormalization constants by Numerical Stochastic Perturbation Theory (NSPT) [3]. A first motivation is a fundamental one: since in principle the proof of multiplicative renormalization has been given in perturbation theory, one should regards an independent perturbative confirmation of non-perturbative results as a precious piece of information. There are of course also more practical motivations, and the quark mass computation is once again a good example. Many groups are involved in the same computation, adopting many (different) regularizations, with many (different) systematic effects to control. We aim at providing high loops results for different regularizations, with a good control on systematics. In the following we will focus the regularization scheme defined by tree level Symanzyk gluonic action and two degenerate $\left(n_{f}=2\right)$ Wilson fermions. Emphasis will be on methodology, for which we claim a very good control on all the systematics at three loop level, with the case of the scalar current taken as an example (and the quark mass always kept as the conceptual background). Actual results will be issued in a future publication.

In order to claim good control on systematics, each approach to the computation of renormalization constants has to face several issues:

- A perturbative computation has of course to face truncation errors, i.e. the feasibility of high loop computations should be addressed.

- Most renormalization schemes are defined in the massless limit. In all these cases one should reach the chiral limit, which in non-perturbative computations is obtained from an extrapolation procedure.

- The continuum limit should be computed.

- In many cases, the renormalization scheme is defined in the infinite volume; thus, finite size effects should be kept under control.

While the first issue only pertains to perturbative computations, all the others are a common problem for both perturbative and non-perturbative computations. 


\section{The control of systematics}

We now proceed to address each source of systematics in our NSPT computations.

\subsection{We can go to high orders}

Why are we able to reach high orders? The very first reason is the use of NSPT [4]. Still, in any NSPT computations, one has to deal with numerical accuracy problems (we can naively think of NSPT as a perturbative MonteCarlo). In view of this, disentangling logarithmic contributions would require a terrific precision.

A popular scheme for renormalization on the lattice is the RI'-MOM scheme. For quark bilinears (like the scalar current) one has to start from Green functions constructed as expectation values computed on external quark states at fixed momentum $p$, from which one then obtains vertex functions by amputation $(S(p)$ is the quark propagator)

$$
G_{\Gamma}(p)=\int d x\langle p|\bar{\psi}(x) \Gamma \psi(x)| p\rangle \quad \Gamma_{\Gamma}(p)=S^{-1}(p) G_{\Gamma}(p) S^{-1}(p) .
$$

The quark field renormalization constants has to be computed from the condition

$$
Z_{q}(\mu, g)=-i \frac{1}{12} \frac{\operatorname{Tr}\left(\not p S^{-1}(p)\right)}{p^{2}} .
$$

After projecting on tree-level structure, one gets renormalization conditions that read

$$
O_{\Gamma}(p)=\left.\operatorname{Tr}\left(\hat{P}_{O_{\Gamma}} \Gamma_{\Gamma}(p)\right) \quad Z_{O_{\Gamma}}(\mu, g) Z_{q}^{-1}(\mu, g) O_{\Gamma}(p)\right|_{p^{2}=\mu^{2}}=1 .
$$

The big advantage of this scheme is that the relevant anomalous dimensions are known at three loop [5]. Being RI'-MOM regulator independent, it is possible to use this information in constructing the renormalization constants we are interested in, e.g.

$$
Z_{q}(\hat{\mu})=1+\sum_{n>0} d_{n} \alpha_{0}^{n}+F(\hat{\mu}) \quad d_{n}=\sum_{i=0}^{n} d_{n}^{(i)} L^{i}
$$

In the previous formula $L$ stands for $\log (a \mu)$, while $F(\hat{\mu})$ entails the irrelevant effects. From the definition of the anomalous dimension, one can get for example for $Z_{q}$

$$
\begin{aligned}
Z_{q}(\hat{\mu}) & =1+Z_{q}^{(1)} \alpha_{0}+\left[Z_{q}^{(2)}-2 \gamma_{q}^{(2)} L\right] \alpha_{0}^{2}+ \\
& +\left[Z_{q}^{(3)}-\left(4 \gamma_{q}^{(2)} K_{1}+2 \gamma_{q}^{(3)}+2 \gamma_{q}^{(2)} Z_{q}^{(1)}\right) L+4 \beta_{0} \gamma_{q}^{(2)} L^{2}\right] \alpha_{0}^{3} .
\end{aligned}
$$

Here $\gamma_{q}^{(i)}$ are the perturnative orders of the quark field anomalous dimension (with the big advantage that $\gamma_{q}^{(1)}=0$ in Landau gauge). The $K_{i}$ are coefficients entering the expansion in $\alpha_{0}$ of the renormalized coupling in which $\gamma$ has been computed (e.g. $\left.\alpha(\mu)=\alpha_{0}-\left(2 \beta_{0} L-K_{1}\right) \alpha_{0}^{2}+\ldots\right)$.

\subsection{We can stay in the chiral limit}

Staying in the chiral limit in perturbation theory simply amounts (for Wilson fermions) to the knowledge of the critical mass. The latter can be computed from the inverse quark propagator

$$
a \Gamma_{2}\left(\hat{p}, \hat{m}_{c r}, \beta^{-1}\right)=a S\left(\hat{p}, \hat{m}_{c r}, \beta^{-1}\right)^{-1}=i \hat{p}+\hat{m}_{W}(\hat{p})-\hat{\Sigma}\left(\hat{p}, \hat{m}_{c r}, \beta^{-1}\right)
$$


by recognizing the component along the identity $\left(\hat{\Sigma}_{c}\left(\hat{p}, \hat{m}_{c r}\right)\right.$ in the expansion of $\hat{\Sigma}\left(\hat{p}, \hat{m}_{c r}, \beta^{-1}\right)$ (in our notation the hat denotes dimensionless quantities and in particular $\hat{p}=p a$ )

$$
\hat{\Sigma}\left(\hat{p}, \hat{m}_{c r}, \beta^{-1}\right)=\hat{\Sigma}_{c}\left(\hat{p}, \hat{m}_{c r}, \beta^{-1}\right)+\hat{\Sigma}_{V}\left(\hat{p}, \hat{m}_{c r}, \beta^{-1}\right)+\hat{\Sigma}_{o}\left(\hat{p}, \hat{m}_{c r}, \beta^{-1}\right) .
$$

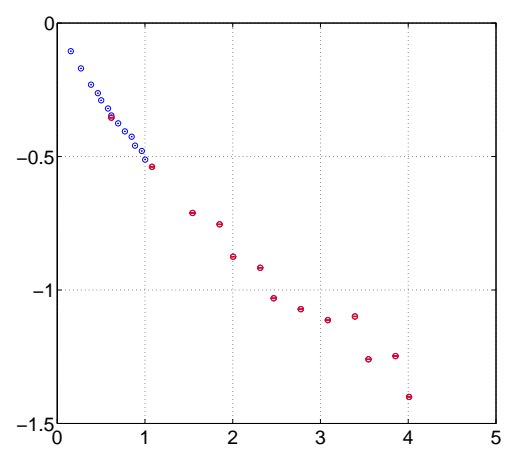

Figure 1: 1-loop $\hat{\Sigma}_{c}\left(\hat{p}, \hat{m}_{c r}\right)$ vs $(a p)^{2}$.

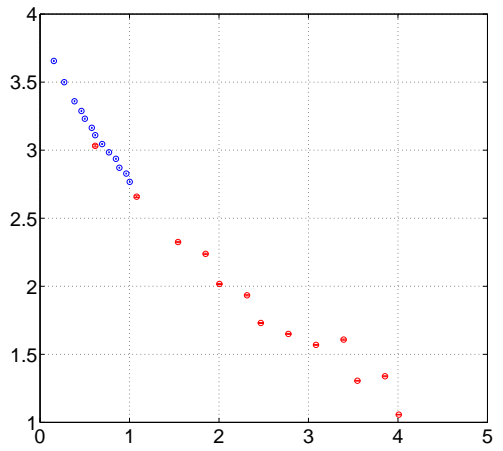

Figure 2: 3-loop $\hat{\Sigma}_{c}\left(\hat{p}, \hat{m}_{c r}\right)$ vs $(a p)^{2}$.

Since 1-loop and 2-loop orders are known [6], we plug their values as counterterms in our computations (see Fig. 1). At 3-loop we get instead a novel result (see Fig. 2): the 3-loop critical mass for the scheme defined by $n_{f}=2$ Wilson fermions and tree level Symanzik gluonic action. In Fig. 1 and 2 we plot data from both $32^{4}$ (blue) and $16^{4}$ (red) lattices.

\subsection{We can get the continuum limit}

We take again the quark field renormalization contant as an example. Suppose one has taken into account the critical mass (so that we are in the chiral limit); one can then tackle the (vector) component along the $\gamma$ matrices, $\hat{\Sigma}_{V}\left(\hat{p}, \hat{m}_{c r}, \beta^{-1}\right)$ in eq. (2.7). Given the hypercubic symmetry of the lattice regularization, we can make the ansatz

$$
\hat{\Sigma}_{V}=i \sum_{\mu} \gamma_{\mu} \hat{p}_{\mu}\left(\hat{\Sigma}_{V}^{(0)}+\hat{p}_{\mu}^{2} \hat{\Sigma}_{V}^{(1)}+\hat{p}_{\mu}^{4} \hat{\Sigma}_{V}^{(2)}+\ldots\right) .
$$

The $\hat{\Sigma}_{V}^{(n)}$ only depend on scalar invariants. Once one has subtracted the relevant logarithms (as dictated by the expansion of the anomalous dimension), we are left with quantities ${ }^{1}$ we can fit to

$$
\hat{\Sigma}_{V}^{(n)}=\alpha_{1}^{(n)} 1+\alpha_{2}^{(n)} \sum_{v} \hat{p}_{v}^{2}+\alpha_{3}^{(n)} \sum_{v} \hat{p}_{v}^{4}+\alpha_{4}^{(n)} \sum_{v \neq \rho} \hat{p}_{v}^{2} \hat{p}_{\rho}^{2}+\mathscr{O}\left(a^{6}\right)
$$

The only finite contribution in the continuum limit is $\alpha_{1}^{(0)}$. We can get it by fitting our data to the previous formula. In Fig. 3 we show an example of such a fit (at 1-loop; keep in mind that there is no $\log$ in this case). The analytical result is known [7] and, as one can see, it is correctly reproduced. Notice that the point associated to the lowest momentum is missed by the fit (actually, it has not even been taken into account). In Fig. 4 we present data from both $32^{4}$ (blue) and $16^{4}$ (red) lattices. Notice that the point associated to the lowest momentum appears to be problematic also in the case of $16^{4}$ lattice.

\footnotetext{
${ }^{1}$ With a slight abuse of notation we use $\hat{\Sigma}_{V}^{(0)}$ for both the log-subtracted and the log-unsubtracted quantity.
} 


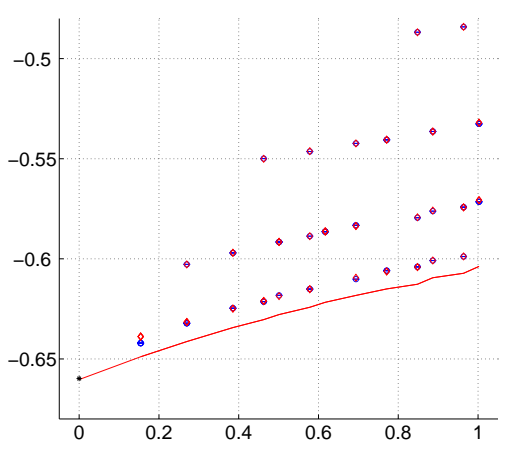

Figure 3: 1-loop $Z_{q}$ vs. $(\text { ap })^{2}$ on $32^{4}$

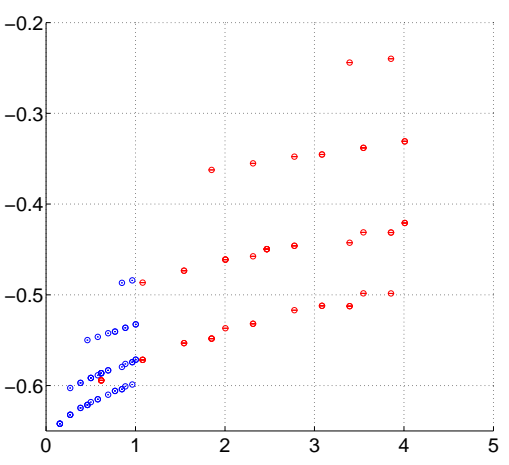

Figure 4: 1 -loop $Z_{q}$ vs. $(\text { ap })^{2}$ on both $32^{4}$ (blue) and $16^{4}$ (red).

\subsection{We can get the infinite volume limit}

As we pointed out in [3], things get worse when one inspects quantities for which an anomalous dimension is in place. The very first case we reported in the case of Wilson gluonic action was just the scalar current. Much the same holds in the present case of tree level Symanzik action. Our master formula (2.3) at 1-loop reads

$$
Z_{q}^{(1)}-Z_{s}^{(1)}=O_{s}^{(1)}-\gamma_{s}^{(1)} L
$$

No $\log$ comes from $Z_{q}$, while $\gamma_{s}^{(1)}$ is the 1-loop anomalous dimension for the current. Fig. 5 summarizes all the relevant information for the problem at hand. The red curve is a fit discarding just the point associated to the lowest momentum and completely misses the right result. The black curve is a fit discarding a wider interval in the IR; it succeeds in getting the analytical result. Fig. 6 shows how this can be ascribed to a finite size effect: the unsubtracted quantity $O_{s}^{(1)}$ is plotted for both a $32^{4}$ (blue) and a $16^{4}$ (red) lattice. One can recognize just the same pattern reported in [3] for the case of the simple plaquette action.

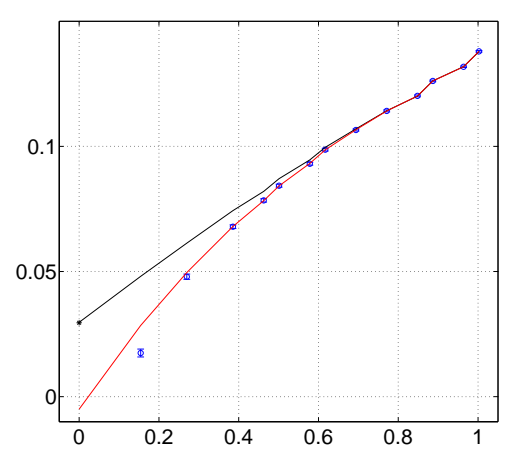

Figure 5: $Z_{q}^{(1)}-Z_{s}^{(1)}$ vs. $(a p)^{2}$ on $32^{4}$; refer to eq. (2.10).

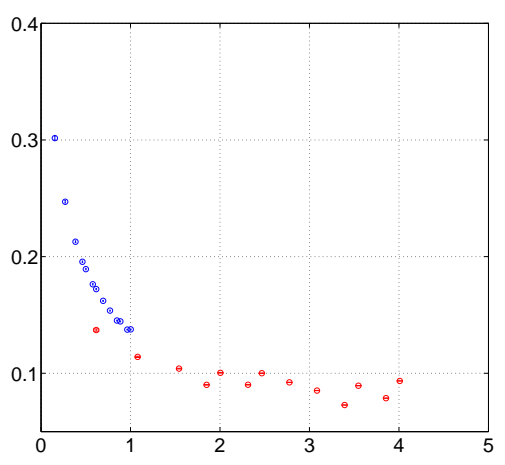

Figure 6: $O_{s}^{(1)}$ vs. $(a p)^{2}$ on both $32^{4}$ (blue) and $16^{4}$ (red); refer to eq. (2.10)

A solution to the problem has been put forward and shown to be effective in [8]. On dimensional grounds, one looks for a dependence on $p L$ and sums and subtracts the infinite volume result 
one is interested in. This defines the finite size error $\Delta \hat{\Sigma}_{V}^{(n)}(\hat{p}, p L)$

$$
\begin{aligned}
\hat{\Sigma}_{V}^{(n)}(\hat{p}, p L) & =\hat{\Sigma}_{V}^{(n)}(\hat{p}, \infty)+\left(\hat{\Sigma}_{V}^{(n)}(\hat{p}, p L)-\hat{\Sigma}_{V}^{(n)}(\hat{p}, \infty)\right) \\
& =\hat{\Sigma}_{V}^{(n)}(\hat{p}, \infty)+\Delta \hat{\Sigma}_{V}^{(n)}(\hat{p}, p L),
\end{aligned}
$$

so that a better formula to fit is

$$
\hat{\Sigma}_{V}^{(n)}(\hat{p}, p L)=\alpha_{1}^{(n)} 1+\alpha_{2}^{(n)} \sum_{v} \hat{p}_{v}^{2}+\alpha_{3}^{(n)} \sum_{v} \hat{p}_{v}^{4}+\alpha_{4}^{(n)}\left(\sum_{v} \hat{p}_{v}^{2}\right)^{2}+\Delta \hat{\Sigma}_{V}^{(n)}(\hat{p}, p L)+\ldots
$$

This is a useful formula once we realize that in first approximation we can look for the $\hat{p}=0$ value for $\Delta \hat{\Sigma}_{V}^{(n)}(\hat{p}, p L)$ (we neglect finite $a$ corrections on top of finite $V$ corrections). We are thus only left with a function of $p L$. This means that we can fit the same correction for the same $\left(n_{1}, n_{2}, n_{3}, n_{4}\right)$ tuple on different lattice sizes:

$$
\Delta \hat{\Sigma}_{V}^{(n)}(\hat{p}, p L) \sim \Delta \hat{\Sigma}_{V}^{(n)}(p L) \quad p_{\mu} L=\frac{2 \pi n_{\mu}}{L} L=2 \pi n_{\mu}
$$

It is useful to go back and inspect again Fig. 3 and Fig. 4: basically, we are saying that the error on the points associated to the lowest momenta - corresponding to the same $(1,1,1,1)$ tuple is in first approximation the same on both $32^{4}$ (blue points) and $16^{4}$ (red points).

\section{Current status of our computations}
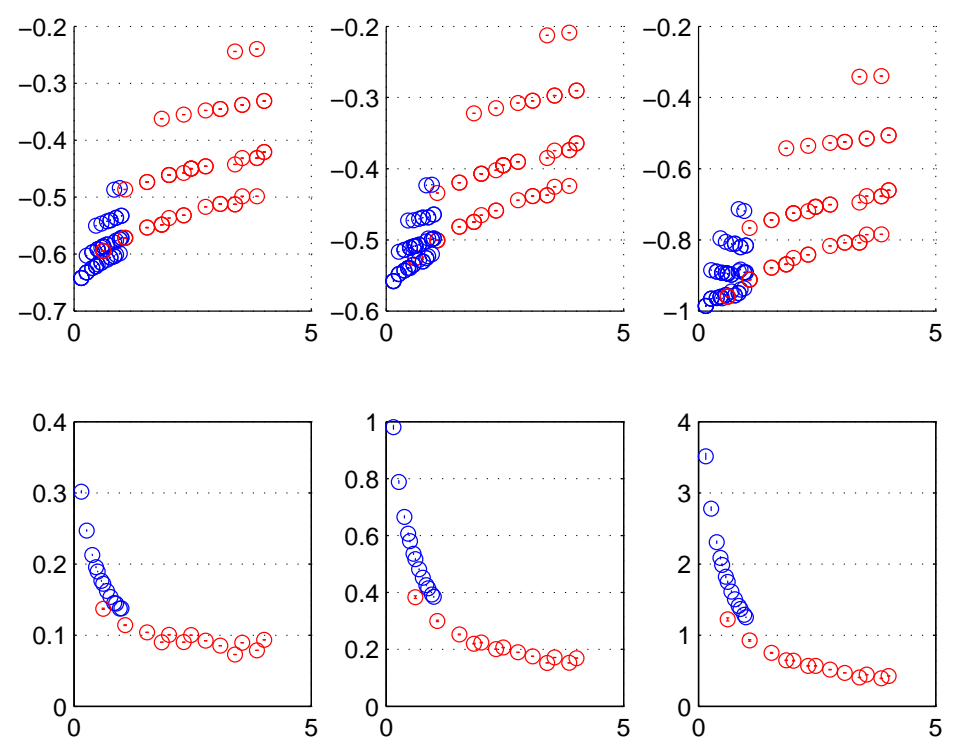

Figure 7: One, two and three loop data for $Z_{q}$ and $O_{s}$. These are unsubtracted data (i.e. logarithms are still to be subtracted). Data for both $32^{4}$ (blue) and $16^{4}$ (red). 
In Fig. 7 we plot our data for the renormalization of the quark field and the scalar current. At the time of the conference we had in our hands only the final data for two lattice sizes $\left(32^{4}\right.$ and $16^{4}$ ). In the meantime data for $24^{4}, 20^{4}$, and $12^{4}$ have been collected. We are currently in the final stage of our analysis, i.e. we are fitting the whole set of data to formulas like eq. (2.12).

\section{Acknowledgements}

This work is supported by I.N.F.N. under the research project MI11 and by the Research Executive Agency (REA) of the European Union under Grant Agreement number PITN-GA-2009238353 (ITN STRONGnet).

\section{References}

[1] For analytical two loops computations of lattice renormalization constants see A. Skouroupathis, H. Panagopoulos, Phys. Rev. D76 (2007) 094514 [arXiv:0707.2906 [hep-lat]]; A. Skouroupathis, H. Panagopoulos, Phys. Rev. D79 (2009) 094508 [arXiv:0811.4264 [hep-lat]].

[2] For a discussion on this issue see for example B. Blossier et al. [ European Twisted Mass Collaboration ], JHEP 0804 (2008) 020 [arXiv:0709.4574 [hep-lat]].

[3] F. Di Renzo, V. Miccio, L. Scorzato and C. Torrero, Eur. Phys. J. C51 (2007) 645-657 [hep-lat/0611013].

[4] NSPT was first introduced in F. Di Renzo, E. Onofri, G. Marchesini and P. Marenzoni, Nucl. Phys. B 426 (1994) 675 [arXiv:hep-lat/9405019]. For a review see F. Di Renzo, L. Scorzato, JHEP 0410 (2004) 073 [hep-lat/0410010].

[5] J. A. Gracey, Nucl. Phys. B 662, 247 (2003) [arXiv:hep-ph/0304113].

[6] A. Skouroupathis, M. Constantinou, H. Panagopoulos, Phys. Rev. D77 (2008) 014513 [arXiv:0801.3146 [hep-lat]].

[7] S. Aoki, K. i. Nagai, Y. Taniguchi and A. Ukawa, Phys. Rev. D 58, 074505 (1998) [arXiv:hep-lat/9802034].

[8] F. Di Renzo, E. -M. Ilgenfritz, H. Perlt, A. Schiller and C. Torrero, Nucl. Phys. B831 (2010) 262-284 [arXiv:0912.4152 [hep-lat]]. F. Di Renzo, E. -M. Ilgenfritz, H. Perlt, A. Schiller and C. Torrero, Nucl. Phys. B842, 122-139 (2011) [arXiv:1008.2617 [hep-lat]]. 